\title{
PRISM-Process Reengineering Integrated Spiral Model: An Agile Approach to Business Process Reengineering (BPR)
}

\author{
Bassam Hussein ${ }^{1}$, Mohamad Hammoud ${ }^{1}$, Hassan Bazzi ${ }^{1} \&$ Amin Haj-Ali ${ }^{1}$ \\ ${ }^{1}$ Lebanese International University, Beirut, Lebanon \\ Correspondence: Bassam Hussein, Lebanese International University, Beirut, Lebanon. Email: \\ bassam.hussein@liu.edu.lb \\ Received: June 17, 2014 \\ Accepted: August 1, $2014 \quad$ Online Published: September 25, 2014 \\ doi:10.5539/ijbm.v9n10p134 \\ URL: http://dx.doi.org/10.5539/ijbm.v9n10p134
}

\begin{abstract}
Over the past two decades, business process reengineering (BPR) has become a popular approach to improve the efficiency and effectiveness of organizations. An examination of relevant BPR literature reveals that available BPR models that have been widely in use have some serious limitations and fail to take into consideration human factors and change management aspects. Both academic researchers and industrial practitioners have acknowledged the existence of this theoretical gap. This article proposes a BPR model that is named process reengineering integrated spiral model (PRISM). PRISM is a systematic agile model that would improve the chances for organizations to successfully carry out BPR initiatives and projects.
\end{abstract}

Keywords: Business process reengineering (BPR), BPR models, organizational change, change management, human factors, risk management

\section{Introduction and Overview}

Both academic researchers and industrial practitioners have introduced their own respective definition of BPR. "No matter how BPR is defined, its main goal is to redesign business process" (Yin, 2010). Kontio (2007) provides an operational definition of BPR as an "approach where processes are developed to maximize an organization's potential". The development of high level models of the business process of organizations can help in understanding how the processes relate to each other. Processes direct teams to fulfill their objectives, such as effectively and efficiently delivering solutions according to users' needs. In an active and constantly changing environment, processes must be agile to cover these changes that are inevitable in a highly globalized market. The process should:

- Enable intra-team communication and coordination,

- Provide good visibility into progress,

- Foster productivity, creativity, and innovation,

- Beholistic and reflect the actual work done.

This means that "the process must be easily maintained, sustained and constantly improved as the organization learns, adapts and evolves" (Hussein, 2008). This article will give an overview of a new agile BPR model referred to as PRISM, will illustrate the fundamental principles and concepts of PRISM, and will show how this approach differs from the modeling methods traditionally used in BPR.

The potential application of PRISM to BPR will be considered and focused on. There will be a thorough discussion about the distinctive features of PRISM in comparison to the traditional BPR models. The characteristics of those models which are similar to the PRISM approach will be highlighted with emphasis on how PRISM can overcome some of their potential problems when carrying out reengineering projects using such models. The relationship between PRISM and agile BPR, the core of this research, will be given special attention and discussed thoroughly.

\section{Process Reengineering Integrated Spiral Model (PRISM)}

Currently, several approaches and models exist and are used for the implementation of BPR (Chi-Kuang \& Cheng-Ho, 2008). However, it has been reported that most of the business organizations which have carried out BPR initiatives followed a traditional approach by using conventional linear life cycle models. Most of the 
existing BPR models were inspired by traditional software development and engineering which have always been criticized for the inconsistency and the variation in their stages. In such approaches, the reengineering effort is broken down into phases where the output of one phase serves as the input to the next. Both the diagnosing and transforming phases must be carried out before any implementation is attempted providing little agility (Hussein et al., 2014). However, software engineering adapted a more agile approach in recent years to overcome the limitations of the traditional models. The spiral model was introduced as a software development and engineering model by Boehm in 1988 to deal with recognized issues with the original process models of the software life cycle, in particular the waterfall model and similar sequential models. The spiral life cycle grew spontaneously from the need to provide an agile and iterative development process. This model provides a well-grounded basis for the PRISM approach as it has been practiced and proven to be successful in the development of large-scale systems. The PRISM approach, depicted in Figure 1, follows a similar path as Boehm's software engineering spiral model and consolidates all of the BPR phases.

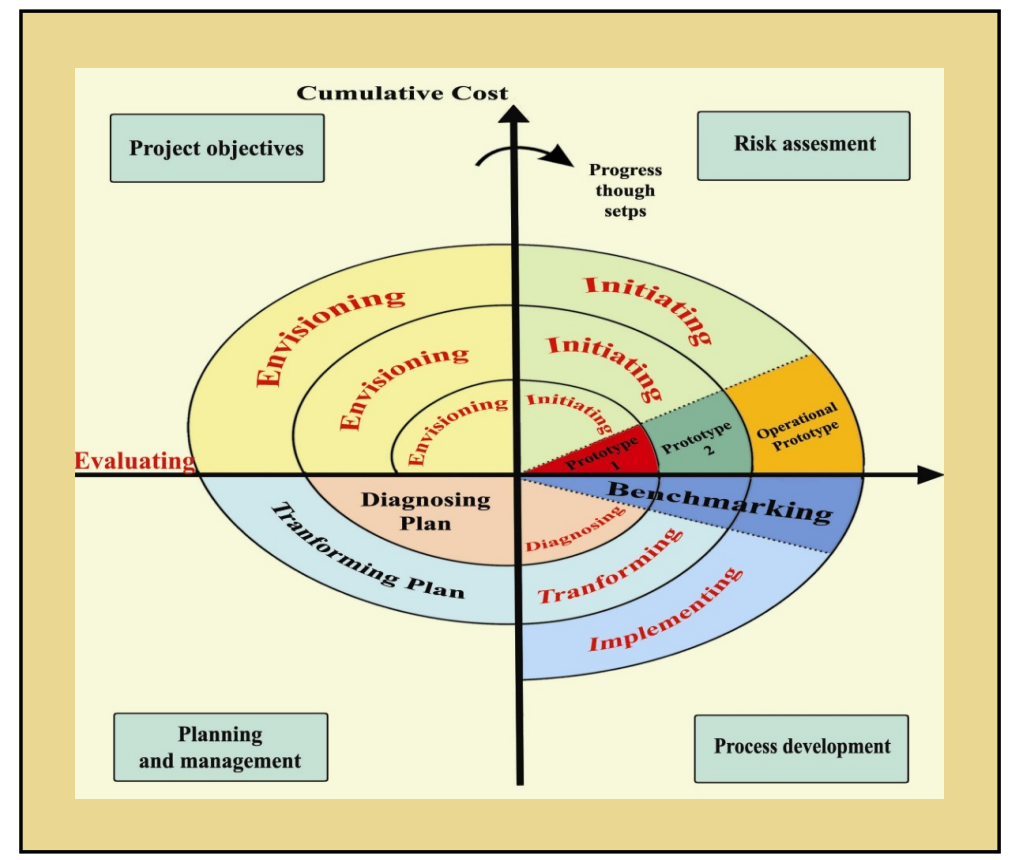

Figure 1. The PRISM approach

The radial dimension, shown in Figure 1, corresponds to the cumulative cost incurred in fulfilling the stages to date, and the angular dimension corresponds to the progress made in completing each cycle of the spiral. The model reflects the inherent concept that each cycle requires a progression which covers the exact succession of steps, for each component of the process in question and for each of its degrees of refinement, from the envisioning phase down to the evaluating one. Each cycle involves traversing through four quadrants. These quadrants are identified as follows:

Project Objectives: Objectives are determined, possible constraints and obstacles for the cycle are identified and alternative approaches are weighed in this quadrant of the model.

Risk Assessment: The second quadrant is a risk analysis and evaluation of alternatives for the cycle. Possible alternatives are examined by the BPR team, and associated risks or problems are identified. Risks resolutions and responses are evaluated and weighed in consideration of project progress. Prototyping is used at this step as a tool to clarify and evolve needs.

Process Development: In this quadrant, the detailed requirements of the process are determined and reengineering is performed. The next level process is verified in this step.

Planning and Management: The process user is given an opportunity to analyze the results of the version created in the process reengineering step and to offer feedback to the process engineer. Planning for the next phases is performed at this step of the model. Lessons learned and Retrospectives are also an important output at 
the end of this quadrant.

The PRISM approach represents a new paradigm for assembling, exposing, organizing and accessing process information. As with all innovations, process users are rarely successful in formulating their needs in addition to the process requirements at early stages in the BPR life cycle. The process objectives and usability are developed along with the evolving users' apprehension of their actual needs. As the process capability evolves, users become more cognizant of further needs and are able to define revised priorities for the characteristics to be admitted into the process. Process stakeholders, who are mainly interested in business aspects, such as enhancing business operations, reducing process life-cycle costs, and incorporating the process into the current infrastructure, render a strategic life cycle-oriented outlook. They help build business objectives and capture the process constraints. The process end-user view constitutes the functional user. Process features originated from end-users; the latters also offer perceptivity into the transformation and implementation details in the reengineering project. Features are the foundation of agile methodologies and are developed within short time-boxes commonly referred to as iterations, cycles or sprints.

The PRISM approach exhibits a practical advantage in the fact that it provides the opportunity of the development of partial specifications which are neither uniform nor rigorous at the beginning. These partial specifications must be analyzed to unveil omitted requirements that can then be addressed in cooperation with the process stakeholders. The approach also uses prototyping as a risk-reducing strategy into the development process. This leads to an improved understanding of the requirements and desired reengineering approaches. An important feature of PRISM is that each cycle is accomplished by an evaluation review involving the BPR team and primary people involved with the process. This review covers all processes reengineered during the previous cycle, which includes the plans for the next cycle and the resources needed to execute them. The main objective of the review is to ensure that all stakeholders (albeit end-users or others) are mutually devoted to the plan and the tactics for the subsequent phase. In addition, it is a reflection of what was done right so that this is adopted and embraced and what did not work well so that it is avoided and not repeated.

PRISM requires the iteration of process reengineering activities at an applicable level of refinement and progression which is fully aligned with agile methodologies. The go alis to institute the most adept solutions at the given level of analysis, via exploring different alternatives. The traitof the process is qualified by more analysis than synthesis at the outset, and more synthesis than analysis at the conclusion. The plans for completing successful phases include the division of the process into increments for consecutive reengineering or components to be reengineered by single departments/units or individuals of the organization. For the latter case, one can picture an array of parallel spiral cycles, one for each component of the process, adding additional dimension to the concept of PRISM presented in Figure 1. For example, separate spirals can be evolving for separate process components or increments. Thus, the "evaluation review and commitment" step may range from an individual walk-through of the transformation of a single process component to a major diagnosis re-appraisal involving key stakeholders such as top management, BPR team, process users, and customers.

An important characteristic of PRISM is its ability to be applied to initiate a BPR effort of a certain process or continue a BPR project to improve a process. In either case, the spiral starts by the hypothesis that a particular process could be reengineered and improved through BPR. The spiral process then evolves into testing this hypothesis. If the hypothesis fails the test, at any point in time, the spiral is terminated. Otherwise, the spiral concludes with the implementation of the reengineered process, and the hypothesis is tested this time by observing the effect on the operational mission. Experience with the operational mission leads usually to further hypotheses about process improvements, and a new improvement spiral is initiated to test the hypothesis. The spiral model implicitly defines the initiation, termination, and iteration of the tasks as well as the processes of previous cycles. PRISM adopts an approach in which activities are initiated concurrently. These activities vary in effort counting on the progress of the project. At the outset, iterations are more process specification intensive and whereas at the conclusion iterations are more process implementation and evaluation intensive.

\section{PRISM's Relationship to the Variables of Successful BPR Models}

Most of the existing BPR models consist of the following six essential phases: envisioning, initiating, diagnosing, transforming, implementing and evaluating. Although these are the same phases that are included in PRISM; however, the conventional paradigm considers a serial rather than a concurrent approach to reengineering. All these phases are isolated from each other and normally failures will not appear until the implementing stage or even later thus increasing unwarranted costs significantly. PRISM provides an integrated and agile environment for these stages in the sense that it is a single process rather than the traditional lifecycle and the same model can be used for diagnosing and transforming of a process and its subsequent implementation. Moreover, PRISM 
allows the evaluation activity to be performed at the early beginnings of the process reengineering project with minimum additional work and, consequently, enables the process to respond rapidly to future evolution or change.

The major distinguishing feature of the PRISM approach is that it creates a risk-driven framework to BPR projects rather than a primarily functionality- driven process reengineering model. It incorporates many of the strengths of existing BPR models and resolves many of their difficulties. In appropriate situations, PRISM becomes equivalent to one of the existing BPR models which were presented earlier in this thesis. In other situations, it provides guidance on the best mix of existing approaches to a given BPR project. For example, if a BPR project has a low risk in certain areas such as not meeting stringent performance requirements, and if it has high risk in areas such as budget and schedule predictability and control, then these risk considerations drive PRISM into equivalence to any of the sequential and traditional BPR models.

A model that does not restrict the BPR team to any particular course of action, but rather allows the reengineering project all the flexibility to develop is a key element of any successful BPR effort (Stergiou \& Johnson, 2004). The PRISM approach acknowledges and accepts that a process is in a state of evolution without pre-supposing a particular change pattern. It provides a roadmap to the reengineering team where a variety of tools, methodologies and techniques may be used at any stage of the BPR life cycle without restrictions. Under this paradigm, PRISM becomes a set of principles that drive process evolution within the guidelines and boundaries of BPR. These factors are crucial in initiating, implementing and deploying BPR in business organizations. In addition, the evolutionary nature of PRISM can extend to business processes as the organization becomes more capable and willing to support it with more enabling structures, procedures and policies.

Effective communication is considered a major key to successful BPR related change efforts. Communication is needed throughout the change process at all different levels and for all potential audience. Communication between the process engineers and the process users in traditional BPR approaches is typically minimal and often difficult, fostering misunderstanding and resentment among them. This may often result in dysfunctional conflict among stakeholders and team members. According to a managing change survey conducted by KPMG,

Only one-third of the respondents reported that their direct managers kept employees well informed of what change was taking place, when the change would occur, and what areas of the organization would be affected. In addition, very few respondents, seventeen percent, stated that their employees have a clear understanding of what BPR is and how it would affect their jobs (Hussein, 2008).

The aim of PRISM is to give the users a more active role in the BPR effort, thereby preventing problems such as poorly understood requirements and eventual user sabotage. Using the PRISM approach encourages intensive communication among all members involved in the reengineering of a certain process due to the fact that the phases of PRISM are carried out concurrently and in an agile evolutionary manner.

It iswidely accepted and acknowledged that business processes cannot be reengineered in a rush. Improving the reengineered process continuously represents an extremely vital part in the success of every reengineering. According to Vakola and Rezgui (2000), the whole BPR activity is a successive and ongoing process in itself. The first step in this activity is monitoring and controlling the progress and the results of the reengineering effort. The progress monitoring consideration is covered by the angular dimension of PRISM which represents the progress through steps. As for monitoring the results, this is handled by the continuous evaluation which is done at the end of each cycle within PRISM. Therefore, continuous improvement of process performance is ensured through these explicit mechanisms which are deployed by PRISM. Consequently, PRISM can be used as an agile approach to handle the various problems encountered during BPR initiatives and continuously improve the process.

\subsection{Main Features of PRISM}

PRISM has many unique properties that overcome the limitations, issues and problems which exist in most of the traditional BPR models. The major characteristics of PRISM, which are considered and discussed later, will focus on PRISM's integrative approach, evolutionary nature, and its adherence to the concepts of risk management, prototyping, benchmarking and continuous improvement all of which are crucial in carrying out business activities nowadays.

\subsection{Integrative Approach}

According to several BPR researchers, it is widely believed that there is a serious need for an integrated approach to BPR. An integrated BPR model which permits the modeling of business processes in an 
organization at various levels with flexibility and agility is very desirable. Ideally, the same model can be used to model processes at a very high level, giving a global view of how processes relate to one another, as well as to model low level processes in great details, allowing these processes to be fine-tuned for maximum operational efficiency and organizational effectiveness. Al-Mashari and Zairi (2000) argue that "BPR may be combined with evolutionary process changes within the same initiatives or integrated into process management by pulling tools from a variety of process change approaches to build hybrid process design and implementation techniques". Based on this argument, PRISM meets this requirement since it goes beyond the limitations of existing non-integrative BPR models and presents BPR in an integrated fashion. The major strength of PRISM is its holistic and systematic approach which integrates BPR with the principles of prototyping, benchmarking, risk management, continuous improvement and dynamic modeling. These principles are used in PRISM's reengineering process and contribute significantly to the success of the BPR project and to the support of the process throughout the reengineering stages as it is based on agile methodologies which are highly flexible and practical.

\subsection{Evolutionary Agile Nature}

A BPR agile model is a structured approach to identify, analyze, and diagnose process problems of organizational trait. An agile model is initially used to analyze the business processes, and then, to evaluate the experimental outcomes with alternative solutions without actually implementing them (Lin, et al., 2002). The following steps are involved in developing an agile modeling approach: problem formulating, problem conceptualizing, model specifying, model checking, solution finding and solution implementing. There are relatively few examples of using dynamic business process models available in the literature (Hlupic, 2001). In one of the articles related to BPR, authors discovered that over 80 percent of BPR projects used static BPR models (Hussein, 2008). Static models, predominately used in BPR initiatives, are deterministic, and do not allow the evaluation of alternative reengineered processes. The use of business process models is usually riveted around modeling existing business process, without providing a systematic approach for evaluating business process alternatives (Hlupic, 2001). On the other hand, the spiral and evolutionary nature of PRISM provides a natural agile approach for BPR. PRISM supports the evaluation of alternative processes in the second quadrant incorporated within its structure, as illustrated in Figure 1. In addition, the results of the implementing phase are processes that feedback, along with planning, into the first phase. In this way, PRISM supports an ongoing infrastructure of effort that drives the continuing evolution of business processes within organizations. PRISM approach has the ability to subdue the complexities of studying and analyzing processes, and thus, lend a higher level of understanding and improving business processes.

In comparison to static BPR models, PRISM depicts a clearer representation of the physical business process environment and alleviated the display of activities and course of events within a process. The advantage of using this agile model is that it allows the evaluation outcome of a reengineered process prior to it being actually implemented. In addition, PRISM captures the resources and their movements within the dynamic model. Noticeably, a large number of failing BPR projects could be attributed to the fact that over 80 percent of business process change deployments employs static modeling (Hussein, 2008). Moreover, the restrictions enforced by this technique mean that it is impossible to predict the outcome of a changed process; one of the reasons contributing to the failure of BPR. PRISM does enable the analysis and evaluation of changed processes; therefore, it is possible that this technique can help to improve the success rate of BPR initiatives.

BPR suggests radical change and radical rewards, but recent studies show a more effective approach may be found in developing a sound management plan for gradual change. Research has shown that people react much more positively to change that is evolutionary. Evolutionary change is more effective but requires careful design and consistent implementation throughout the various phases of change (Campbell \& Kleiner, 1997). This concept is fundamental to the PRISM approach. The evolutionary nature of PRISM's cycles provides an agile model which promotes periodic changes and updates to the reengineered process on a continuous basis as needed. This contributes to the business strategy by taking into account the changing conditions of external variables, such as competition, customer satisfaction, economic environment, and unpredictable or sudden occurrences in the market place. It also accommodates the needs of people within the organization to adapt to change in a gradual fashion thus minimizing their resistance.

\subsection{Risk Management}

BPR has been both a high-risk and a high-reward proposition. BPR can be very risky as studies show that a large percentage of reengineering efforts fail. Numerous reports in the literature indicate that BPR often misses its mark, failing to deliver the anticipated benefits. Therefore, since reengineering efforts require large investments 
of an organization's resources, special precautions must be taken before embarking on a project. An organization needs to clarify its motives, decide how much change is required and establish the scope of what it is trying to accomplish. Managers frequently get excited to start a project without knowing or understanding the risks involved and end up wasting valuable resources. Risks are potential future occurrences that may significantly impact the outcome of the project. A risk is usually defined in terms of its probability or likelihood, which often can only be estimated subjectively, and its impact. Risk management hinges on the identification and evaluation of risks. This process must be carried out perpetually throughout the reengineering project. For each ominous risk, a plan for a course of counteraction must be furnished. This plan may try to avoid the risk, if possible, mitigate its impact, or accept its results. In the case where avoidance or mitigation has been opted, an individual must assume responsibility for developing avoidance and mitigation strategies, monitoring the risk, and initiating any necessary actions. Similarly, when a risk offers an opportunity, this opportunity must be evaluated and plans prepared to benefit from its occurrences. Risks are logged and tracked in a risk register or any similar tool. The register must be formally and regularly reviewed. However, the keys to successful risk management do not rest in the employment of specific risk management tools, rather in the effective identification of risks, assessment, ownership, and particularly, the development of cost-effective avoidance and mitigation strategies. It must be noted that risk management process should be positive and proactive, value-based and holistic, integrated in processes, inherent in the strategy and total operations, and uninterrupted (Woon et al., 2011). Contingency planning must not be overlooked as well.

Risk management is explicitly included in PRISM as a step in the reengineering process and a means of evaluating each version of the process to determine whether or not reengineering should continue. If the process user decides that any identified risks has great impact with respect to costs and/or, the project may be stopped. For example, if a significant increase in cost or project completion delay is identified during one phase of risk assessment, the process user/engineer may decide that it is more sensible to halt the project, since the increased cost or lengthened schedule may make continuation of the project impractical or unfeasible. Therefore, PRISM provides a continuous risk assessment throughout the implementation process to deal with any risk at its initial state and to ensure the success of the BPR initiative. Properly and adequately anticipating and planning risk management is important for dealing effectively with risks when they first occur. This will focus risk management function from primarily defensive to increasingly offensive and strategic in nature (Woon et al., 2011).

\subsection{Prototyping}

Once the BPR team has a concept of the reengineered process, it can prototype the process to simulate and test it for shortcomings or issues and remedy those before the actual implementation. Prototypes create a basic working process which does not contain all of the requirements desired by the process user, but which works properly and provides the essential initial elements of the process. Prototyping is an iterative process which serves to reduce implementation risks through simulation, pilot processes, modelling, and small-scale implementation. As such, it is also considered as a pillar of agile development. A pilot process, which is a small-scale and fully operational implementation of a new process, may be used to learn how to bring the change and proceed with BPR. Ron Baker, the vice president of enterprise system solutions for Massachusetts Mutual Life Insurance in Hartford, Connecticut, believes that "the concepts of using a pilot phase and prototyping are critical in BPR, especially when dealing with user interfaces and workflow processes" (Hussein, 2008).

The transformation of the reengineered process should not be viewed as the termination of the BPR process, but as a prototype with successive iterations. The metaphor of prototyping aligns the BPR approach with evolutionary process modelling, rapid delivery of results and the better engagement of customers leading to increased chance of their acceptance of the final product or service. Based on this concept, PRISM incorporates prototyping, as depicted in Figure 1, as a risk reduction option at the early stages of the reengineering project. It must be noted that PRISM uses prototyping in the process of going from the evaluating, identifying and resolving risks step into the developing and verifying next level process step. The timing of the prototyping activity between those two steps is extremely crucial due to the fact that the transition at this point is very significant in terms of cost and risk. The process of prototyping is identified as a crucial process within BPR (Yin, 2010).

The notion of prototyping is included in the PRISM approach that focuses on the following distinguishable areas:

Prototyping may be used to rapidly develop sketches of particular processes or components, in the case where the user requirements are not well understood or are difficult to formally determine. This will assist in the 
reengineering process by shifting the focus on those areas of the requirements that need more refinement before implementation are made and thus change later on becomes more difficult to apply.

Prototyping can also be used for risk management functions in the evaluating phase. It serves in the development of processes or components outlines. Candidate processes are those which are crucial to the success of the project, but whose specification or transformation may contain uncertainties. The uncertainty associated with this type of components and processes may represent an actual threat to the success of the project, and hence prototyping is used to explore the feasibility of the project in an area, which could prove to be excessively expensive, or perhaps beyond current capabilities.

Prototyping can also be used as a demonstration tool to illustrate to the client the potential traits and functions of the final process, thus assisting in the economic consideration of the process. Additional benefit is provided when prototyping is used as an effective tool for obtaining early feedback on the role of the process.

\subsection{Benchmarking}

Benchmarking is defined as $\mathrm{s}$ the process of identifying, understanding, and adapting best practices by using scientific evidence to guide performance improvement measures within and among organizations (Kardooni, 2008). A benchmark can be considered to be anything that is used and measured as a point of comparison, reference or as a standard by which service is considered. Benchmarking is an enabler for achieving and maintaining high levels of competitiveness and is the measurement of business performance against the best practices through a continued effort of constantly reviewing processes, practices and methods. When applied to processes, benchmarking highlights the negative gaps in performance, hence, enabling suitable actions to be taken in all areas to maximize the level of performance needed to be the best in the class (Al-Mashari \& Zairi, 2000).

A typical cycle of PRISM begins with the identification of the process to be reengineered, the alternatives for implementing this process, and the constraints imposed on the application of alternatives. The next step is to evaluate the alternatives relative to the objectives and constraints in order to identify the areas of uncertainty that are significant sources of BPR project risks. This may be achieved through benchmarking, as shown in Figure 1, where improvement targets are set through identifying the performance and practices of similar processes. According to a survey carried out by Al-Mashari et al. (2001), it was shown that there is an overall agreement across all surveyed organizations in rating the level of using five approaches of benchmarking in BPR. The findings of this survey are summarized in Table 1. The rating used in this survey is on a scale of 1 , being very low, to 5 , being very high.

Table 1. Ranking of use of benchmarking in BPR

\begin{tabular}{|c|c|c|c|c|c|c|}
\hline \multirow{2}{*}{ Use of benchmarking in BPR } & \multicolumn{2}{|c|}{ Overall } & \multicolumn{2}{|l|}{$U S A$} & \multicolumn{2}{|c|}{ Europe } \\
\hline & Mean & Rank & Mean & Rank & Mean & Rank \\
\hline To identify best practice & & & & & & \\
\hline $\begin{array}{l}\text { performance and process in the } \\
\text { future }\end{array}$ & 3.46 & 1 & 3.44 & 1 & 3.48 & 1 \\
\hline $\begin{array}{l}\text { To highlight areas of change and } \\
\text { prioritize them }\end{array}$ & 3.10 & 2 & 2.91 & 2 & 3.30 & 2 \\
\hline $\begin{array}{l}\text { To identify innovative process } \\
\text { attributes }\end{array}$ & 2.87 & 3 & 2.81 & 3 & 2.93 & 3 \\
\hline To determine process objectives & 2.79 & 4 & 2.69 & 4 & 2.90 & 4 \\
\hline $\begin{array}{l}\text { To make a proper decision about } \\
\text { type of change whether to be } \\
\text { evolutionary or revolutionary }\end{array}$ & 2.49 & 5 & 2.50 & 5 & 2.48 & 5 \\
\hline
\end{tabular}

\subsection{Continuous Improvement}

PRISM is designed to accommodate for process evolution throughout its life cycle, process growth, and changes or modifications of the process. This is achieved through the evaluation phase which is included in every cycle of the spiral and provides for an evolutionary evaluation of the progress of the reengineered process. Continuous improvement involves incremental continuous change through the involvement of functions reengineering the existing process in an evolutionary manner (Dooley \& Johnson, 2001). Many BPR advocates argue that it is 
extremely crucial to integrate BPR with a mechanism that allows for continuous improvement of business processes in order to achieve dramatic gains. Continuous quality improvement (CQI), total quality management (TQM), and ISO 9000 are a few of a wide range of continuous improvement techniques that are available for use with BPR (Hussein, 2008).

Studies conducted by a group of practitioners and researchers suggest that BPR integrated with continuous improvement can achieve better performance (Hussein, 2008). The reason is that no single approach is believed to be suitable for performance improvement at all times (Al-Mashari \& Zairi, 2000). Therefore, some authors like Davenport (1993) and Kelada (1994) advocate that BPR and continuous improvement must be combined on an ongoing integrated management system to ensure the improvements that reengineering brings to organizations. BPR should always be regarded as a successive and ongoing improvement strategy. A rigorous well-constructed analysis must be undertaken to ensure validity after each BPR cycle (Kardooni, 2008).

\section{Conclusion}

This paper described PRISM as a novel agile approach to BPR modelling in a situated and open-ended manner. Based on the concepts of evolutionary modelling, risk management, integration, and many other features, the process of the PRISM approach is the construction of an agile BPR model in a fashion similar to the way in which humans form a mental model of the real world subject.

To sum up, conventional BPR models which formalize the reengineering process and preconceive the possible states in the operational domain may be attractive due to their simplistic linear approach, but may also restrict the flexibility and agility of process engineers in choosing alternatives and their openness to new concerns. In contrast, PRISM is an evolutionary and agile approach where the BPR phases and activities are not performed in a sequential fashion, but rather is a concurrent and parallel one. The PRISM approach constitutes a formalized model for carrying out BPR and helping business organizations to streamline their business processes according to their goals and objectives in a dynamic fashion.

\section{References}

Al-Mashari, M., \& Zairi, M. (2000). Revisiting BPR: a holistic review of practice and development,Business Process Management Journal, 6(1), 10-42. http://dx.doi.org/10.1108/14637150010283045

Al-Mashari, M., Irani, Z., \& Zairi, M. (2001). Business process reengineering: a survey of international experience. Business Process Management Journal, 7(5), 437-455. http://dx.doi.org/10.1108/14637150110406812

Campbell, S., \& Kleiner, B. (1997). New developments in reengineering organizations. Work Study, 46(3), 99-103. http://dx.doi.org/10.1108/00438029710162953

Chi-Kuang, C., \& Cheng-Ho, T. (2008). Developing a process re-engineering-oriented organizational change exploratory simulation system (PROCESS). International Journal of Production Research, 46(16), 446-4482.

Davenport, T. (1993). Process innovation: Reengineering work through information technology. Boston: Harvard Business School Press.

Dooley, K., \& Johnson, D. (2001). Changing the new product development process: Reengineering or continuous quality improvement? Measuring Business Excellence, 5(4), 32-38. http://dx.doi.org/10.1108/EUM0000000006517

Green, D. (2004). Kaizen JIT and TQM compared and contrasted with business process reengineering.

Hlupic, V. (2001). Current trends in business process modeling. International Journal of Simulation Systems Science and Technology, 2(2), 1-4.

Hussein, B. (2008). PRISM: Process Re-engineering Integrated Spiral Model. VDM Verlag, Berlin.

Hussein, B., Chouman, M., \& Dayekh, A. (2014). A project life cycle (PLC) based approach for effective business process reengineering (BPR). Industrial Engineering Letters, 4(6).

Kardooni, S., Haut, E. R., Chang, D. C., Pierce, C. A., Efron, D. T., Haider, A. H., \& Cornwell III, E. E. (2008). Hazards of benchmarking complications with the National Trauma Data Bank: numerators in search of denominators. Journal of Trauma-Injury, Infection, and Critical Care, 64(2), 273-279. http://dx.doi.org/10.1097/TA.0b013e31816335ae

Kelada, W. (1994). Is reengineering replacing total quality? Quality Progress, 27(12), 79-85. 
Kontio, J. (2007). Business process re-engineering: a case study at Turku University of Applied Sciences. Proceedings of European and Mediterranean Conference on Information Systems 2007 (EMCIS2007), 24-26.

Lin, F., Yang, M., \& Pai, Y. (2002). A generic structure for business process modelling. Business Process Management Journal, 8(1), 19-41. http://dx.doi.org/10.1108/14637150210418610

Vakola, M., \& Rezgui, Y. (2000). Critique of existing process re-engineering methodologies: the development and implementation of a new methodology. Business Process Management Journal, 6(3), 238-250. http://dx.doi.org/10.1108/14637150010325453

Woon, L. F., Azizan, N. A., \& Samad, M. F. A. (2011). A strategic framework for value enhancing enterprise risk management. Journal of Global Business and Economics, 2(1), 23-47.

Yin, G. (2010). BPR application. Modern Applied Science, 4(4), 96-101. http://dx.doi.org/10.5539/mas.v4n4p96

\section{Copyrights}

Copyright for this article is retained by the author(s), with first publication rights granted to the journal.

This is an open-access article distributed under the terms and conditions of the Creative Commons Attribution license (http://creativecommons.org/licenses/by/3.0/). 